\title{
Estudo do perfil epidemiológico dos pacientes portadores de tuberculose na Região de Saúde Centro-Sul (RJ) dos casos confirmados entre 2015 e 2019.
}

\section{Study of the epidemiological profile of patients with tuberculosis in the Center-South Health Region (RJ) of confirmed cases between 2015 and 2019.}

Renato Franco Borges ${ }^{1 *}$; Sebastião Jorge da Cunha Gonçalves².

Como citar esse artigo. Borges, R.F;

Gonçalves, S.J.C. Estudo do perfil

epidemiológico dos pacientes portadores de tuberculose na Região de Saúde Centro-Sul (RJ) dos casos confirmados entre 2015 e 2019. Revista de Saúde. 2021 Abr./Jul.; 12 (2): 49-58.

\begin{abstract}
Resumo
Cerca de um terço da população mundial está infectada pelo Mycobacterium tuberculosis e o Brasil é um dos países com maior número de casos no mundo. O objetivo do presente estudo foi desenhar o perfil epidemiológico dos pacientes com tuberculose na Região de Saúde Centro-Sul (RJ) no período de 2015 a 2019, visando a criação de ações para a prevenção da ocorrência de novos casos, diminuindo morbidades e mortalidade, além do gasto com tuberculose pelo SUS de forma local. Trata-se de um estudo transversal, observacional, quantitativo, descritivo, onde os dados foram retirados do Sistema de Informação de Agravos de Notificação - Sinan Net, dos anos de 2015 a 2019. Foram notificados 741 casos de tuberculose no período, onde se analisou as seguintes variáveis: faixa etária, escolaridade, raça, sexo, tipo de entrada, forma dos casos, infecção conjunta com HIV, realização de tratamento supervisionado e situação de encerramento do caso. O perfil mais comum encontrado foi de pacientes com 20 a 39 anos (38,73\%), ensino médio completo (9,04\%), raça branca $(28,20 \%)$, sexo masculino $(69,64 \%)$, caso novo $(82,19 \%)$, forma pulmonar $(89,61 \%)$, infecção pelo HIV (negativo $83,81 \%)$, realização de tratamento supervisionado $(59,92 \%)$ e encerramento por cura $(65,05 \%)$. O perfil mais comum encontrado foi similar ao de estudos realizados em outras cidades e regiões do Brasil. Há ainda muito a melhorar na Região de Saúde Centro-Sul (RJ), embora esteja com números superiores aos índices nacionais para a maioria das variáveis analisadas no estudo.
\end{abstract}

Palavras-chave: Tuberculose; Perfil de saúde; Controle de doenças transmissíveis;

\begin{abstract}
About one third of the world population is infected with Mycobacterium tuberculosis and Brazil is one of the countries with the largest number of cases in the world. The objective of the present study was to draw the epidemiological profile of patients with tuberculosis in the Central-South Health Region (RJ) in the period from 2015 to 2019, aiming to create actions to prevent the occurrence of new cases, reducing morbidities and mortality, in addition to spending on tuberculosis by SUS locally. This is a cross-sectional, observational, quantitative, descriptive study, where the data were taken from the Information System for Notifiable Diseases - Sinan Net, from the years 2015 to 2019.741 cases of tuberculosis were reported in the period, where it was analyzed the following variables: age group, education, race, sex, type of entry, form of cases, joint infection with HIV, conducting supervised treatment and situation of closing the case. The most common profile found was patients aged 20 to 39 years $(38.73 \%)$, complete high school $(9.04 \%)$, white race $(28.20 \%)$, male $(69.64 \%)$, new case $(82.19 \%)$, pulmonary form $(89.61 \%)$, HIV infection (negative $83.81 \%)$, supervised treatment $(59.92 \%)$ and closure due to cure $(65.05 \%)$. The most common profile found was similar to that of studies carried out in other cities and regions of Brazil. There is still much to improve in the Centro-Sul Health Region (RJ), although it has numbers higher than the national indices for most of the variables analyzed in the study.
\end{abstract}

Keywords: Tuberculosis; Health profile; Communicable Disease Control;

\section{Introdução}

Desde os primórdios, a tuberculose aflige a humanidade, de forma que é a doença com um agente específico que mais causa mortes no mundo, sendo 99\% delas em regiões subdesenvolvidas ${ }^{1}$. Em 2018, em todo o mundo, cerca de 10 milhões de pessoas tiveram tuberculose e dessas, 1,5 milhão faleceram².

Cerca de um terço da população mundial está infectada pelo Mycobacterium tuberculosis, e o Brasil é um dos países com maior número de casos no mundo. No ano de 2015, no Brasil, 69.000 pessoas adoeceram com tuberculose, 6.800 pessoas viveram com HIV e desenvolveram tuberculose, 4.500 homens, mulheres e crianças morreram de tuberculose e 1.077 pessoas desenvolveram tuberculose multidrogarresistente ${ }^{3}$.

Um caso novo de tuberculose custa aproximadamente ao Sistema Único de Saúde (SUS) cerca de $\mathrm{R} \$ 293,91$. Os itens que representam maior custo são profissionais da saúde e medicamentos.

\footnotetext{
Afiliação dos autores:

${ }^{1}$ Discente da Universidade de Vassouras, Vassouras, Rio de Janeiro, Brasil, Orcid ID 0000-0001-6133-4301;

${ }^{2}$ Docente da Universidade de Vassouras, Vassouras, Rio de Janeiro, Brasil, Orcid ID 0000-0002-4228-4641;
} 
Entretanto, embora o SUS ofereça o tratamento gratuito, há gastos tangíveis e intangíveis para os pacientes e familiares, alcançando cerca de $48 \%$ da renda com despesas relacionadas à tuberculose, somando um total de $\mathrm{R} \$ 3.119,40$, o que gera um impacto extremamente elevado nas famílias ${ }^{4}$.

A tuberculose é uma doença produzida pelas condições de vida dos indivíduos. Nesse sentido, a diminuição da pobreza e da miséria seria uma solução sobre seus efeitos sobre a saúde das populações. Com base nessa premissa que os programas de controle da tuberculose são criados, determinando um conjunto de ações para o enfrentamento da doença e das condições que favoreçam sua reproduçãos.

O Ministério da Saúde (MS) lançou em 2017, o Plano Nacional pelo Fim da Tuberculose, colocando como meta a redução de casos para um número menor que dez casos por 100.000 habitantes e diminuir a mortalidade para um número menor que uma morte para cada 100.000 habitantes até 2035 , que representaria o fim da tuberculose como problema de saúde pública, assim o próximo passo seria buscar a eliminação da tuberculose, que significa menos de um caso por 1.000.000 de habitantes ${ }^{3}$.

Diversos estudos já abordaram o perfil epidemiológico da tuberculose no Brasil a nível nacional, assim como diversos trabalhos também traçaram o perfil epidemiológico em algumas regiões, mas após pesquisa em diversas plataformas, não foram encontrados estudos da Região de Saúde Centro-Sul (RJ) ou cidades específicas que a compõem.

O objetivo do presente trabalho é desenhar o perfil epidemiológico com as características mais comuns dos pacientes com tuberculose nos últimos cinco anos (2015 a 2019) na Região de Saúde Centro-Sul (RJ), visando a criação de futuras ações para a prevenção da ocorrência de novos casos, diminuição de morbidades e mortalidade, além de diminuir o gasto do SUS com tuberculose de forma local.

\section{Materiais e Métodos}

Trata-se de um estudo transversal, observacional, quantitativo, descritivo, por meio dos casos coletados do Sistema de Informação de Agravos de Notificação - Sinan Net, que são disponibilizados no site do Departamento de Informática do Sistema Único de Saúde (DATASUS) do Ministério da Saúde. A forma de acesso aos dados foi realizada conforme o fluxograma da Figura 1.

Os dados foram consultados em 24 de julho de 2020, em que se retiraram informações acerca do perfil epidemiológico dos pacientes com tuberculose na Região de Saúde Centro-Sul (RJ) de 2015 a 2019, ou seja, dos últimos 5 (cinco) anos disponíveis. A

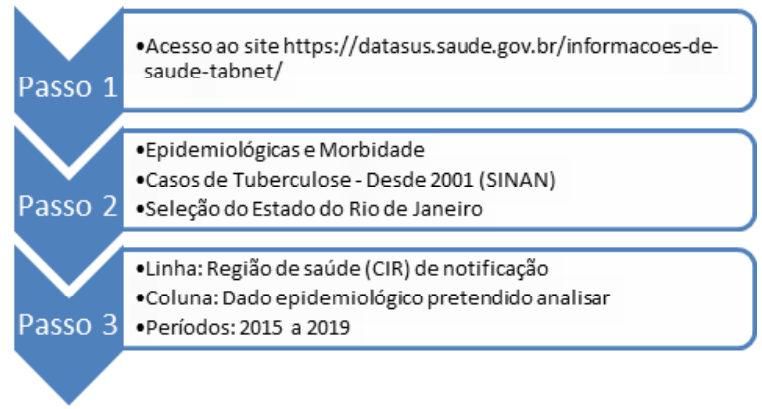

Figura 1. Fluxograma das etapas de acesso ao DATASUS.

população foi composta por 741 (setecentos e quarenta e um) pacientes que tiveram o diagnóstico de tuberculose notificado no Sinan Net.

A Região Centro-Sul (RJ) é composta por 11 municípios citados a seguir: Paracambi, Engenheiro Paulo de Frontin, Mendes, Miguel Pereira, Paty do Alferes, Paraíba do Sul, Areal, Três Rios, Comendador Levy Gasparian, Sapucaia e Vassouras.

As variáveis analisadas foram faixa etária, escolaridade, raça, sexo, tipo de entrada, forma dos casos, infecção conjunta com HIV, realização de tratamento supervisionado e situação de encerramento do caso.

Compararam-se, através de tabelas, as características mais comuns de cada seleção de dados disponíveis, selecionando os que foram mais relevantes para traçar o perfil epidemiológico. Para coleta e organização dos dados, assim como confecção de gráficos, foram utilizados os softwares do pacote Microsoft Office, Word e Excel.

\section{Resultados}

Os dados foram coletados através do Sinan Net (Sistema de Informação de Agravos e Notificação) do Ministério da Saúde em 24 de julho de 2020, onde se constatou um total de 741 notificações de pacientes, conforme Tabela 1, que tiveram tuberculose entre 2015 e 2019 na Região de Saúde Centro-Sul (RJ).

Foram analisadas nove características para traçar o perfil epidemiológico dos 741 pacientes, sendo elas: faixa etária, escolaridade, raça, sexo, tipo de entrada, forma, HIV, TDO (Tratamento Supervisionado) e situação de encerramento.

A Tabela 2A mostra a quantidade de pacientes com tuberculose por faixa etária na Região de Saúde Centro-Sul (RJ), e a Tabela 2B em todo o Brasil. Houve maior percentual de casos na faixa entre 20 e 39 anos, embora na faixa entre 40 e 59 anos o percentual tenha sido muito próximo. Ou seja, um intervalo entre 20 a 59 anos, poupando mais os extremos de idade, menores 
Tabela 1. Casos confirmados de tuberculose por Região de Saúde do Estado do Rio de Janeiro entre 2015 e 2019.

\begin{tabular}{lcc}
\hline Região de Saúde (CIR) de notificação & Casos confirmados & Percentual \\
\hline 33001 Baia da Ilha Grande & 808 & $1,14 \%$ \\
33002 Baixada Litorânea & 1709 & $2,42 \%$ \\
33003 Centro-Sul & $\mathbf{7 4 1}$ & $\mathbf{1 , 0 4 \%}$ \\
33004 Médio Paraíba & 1997 & $2,82 \%$ \\
33005 Metropolitana I & 54994 & $77,89 \%$ \\
33006 Metropolitana II & 5806 & $8,22 \%$ \\
33007 Noroeste & 619 & $0,87 \%$ \\
33008 Norte & 2412 & $3,41 \%$ \\
33009 Serrana & 1518 & $2,15 \%$ \\
Total & 70604 & $100 \%$ \\
\hline
\end{tabular}

Fonte: Ministério da Saúde - Sistema de Informação de Agravos e Notificação - Sinan

de 20 anos e maiores de 59 anos. No Brasil, as faixas etárias mais comuns também são as de 20 a 39 anos e 40 a 59 anos, conforme Tabela 2B.
Quanto à escolaridade, a Tabela 3A mostra os grupos com maiores incidências na Região de Saúde Centro-Sul (RJ) são os da $5^{\mathrm{a}}$ a $8^{\mathrm{a}}$ série incompleta, ensino fundamental completo, ensino médio incompleto e ensino médio completo, totalizando $27,38 \%$ do total de casos. Também há menor incidência nos extremos. Há de se destacar que os dados ainda estão insuficientes para uma análise mais aprofundada, pois $61,40 \%$ dos pacientes notificados não tiveram tal informação incluída na notificação. No Brasil, essa informação também foi ignorada em grande percentual de notificações, mas em menor percentual que a Região de Saúde Centro-Sul (RJ), com 26,81\%, conforme Tabela 3B.

Em relação à raça declarada, a Tabela 4A mostra que há maior incidência na raça branca na Região de Saúde Centro-Sul (RJ), embora também haja um grande número de casos notificados com tal informação ignorada. Apesar de maior parte ser da raça branca, a diferença para o número de casos de pacientes de raça preta e parda não foi considerável, $23,48 \%$ e $22,67 \%$, respectivamente, além de baixo percentual para as raças

Tabela 2A. Faixa etária dos casos de tuberculose na Região de Saúde Centro-Sul (RJ) entre 2015 e 2019.

\begin{tabular}{|c|c|c|c|c|c|c|c|c|c|c|c|}
\hline \multicolumn{12}{|c|}{ Região de Saúde Centro-Sul (RJ) } \\
\hline Faixa etária & $<1$ ano & 1 a 4 & 5 a 9 & 10 a 14 & 15 a 19 & 20 a 39 & 40 a 59 & 60 a 64 & 65 a 69 & 70 a 79 & $80 \mathrm{e}+$ \\
\hline Pacientes & 9 & 5 & 5 & 8 & 43 & 287 & 261 & 41 & 38 & 31 & 13 \\
\hline Percentual & $1,21 \%$ & $0,67 \%$ & $0,67 \%$ & $1,07 \%$ & $5,80 \%$ & $38,73 \%$ & $35,22 \%$ & $5,53 \%$ & $5,12 \%$ & $4,18 \%$ & $1,75 \%$ \\
\hline
\end{tabular}

Tabela 2B. Faixa etária dos casos de tuberculose no Brasil entre 2015 e 2019.

\begin{tabular}{|c|c|c|c|c|c|c|c|c|c|c|c|}
\hline \multicolumn{12}{|c|}{ Brasil } \\
\hline Faixa etária & $<1$ ano & $1 \mathrm{a} 4$ & 5 a9 & 10 a 14 & 15 a 19 & 20 a 39 & 40 a 59 & 60 a 64 & 65 a 69 & 70 a 79 & $80 \mathrm{e}+$ \\
\hline Pacientes & 2108 & 2965 & 2637 & 4913 & 25159 & 207543 & 140440 & 22860 & 15925 & 18430 & 7591 \\
\hline Percentual & $0,46 \%$ & $0,65 \%$ & $0,58 \%$ & $1,09 \%$ & $5,58 \%$ & $46,05 \%$ & $31,16 \%$ & $5,07 \%$ & $3,53 \%$ & $4,08 \%$ & $1,68 \%$ \\
\hline
\end{tabular}

Tabela 3A. Escolaridade dos pacientes com tuberculose na Região de Saúde Centro-Sul (RJ) entre 2015 e 2019.

\begin{tabular}{|c|c|c|c|c|c|c|c|c|c|c|c|}
\hline \multicolumn{12}{|c|}{ Região de Saúde Centro-Sul (RJ) } \\
\hline Escolaridade & 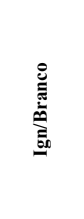 & 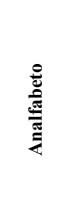 & 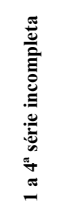 & 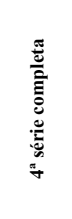 & 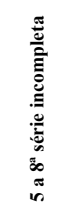 & 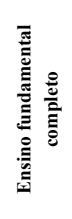 & 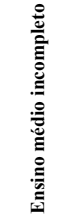 & 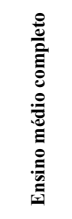 & 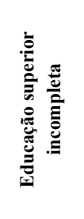 & 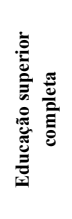 & 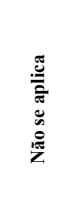 \\
\hline Pacientes & 455 & 7 & 26 & 18 & 52 & 45 & 39 & 67 & 7 & 9 & 16 \\
\hline Percentual & $61,40 \%$ & $0,94 \%$ & $3,5 \%$ & $2,42 \%$ & $7,01 \%$ & $6,07 \%$ & $5,26 \%$ & $9,04 \%$ & $0,94 \%$ & $1,21 \%$ & $2,15 \%$ \\
\hline
\end{tabular}


Tabela 3B. Escolaridade dos pacientes com tuberculose no Brasil entre 2015 e 2019.

\begin{tabular}{|c|c|c|c|c|c|c|c|c|c|c|c|}
\hline \multicolumn{12}{|c|}{ Brasil } \\
\hline Escolaridade & 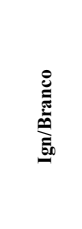 & 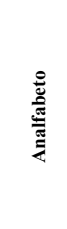 & 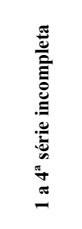 & 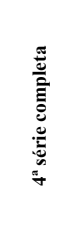 & 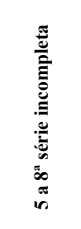 & 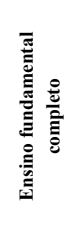 & 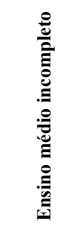 & 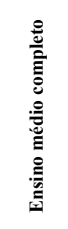 & 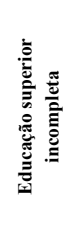 & 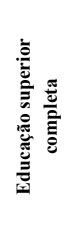 & 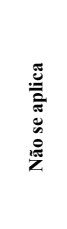 \\
\hline Pacientes & 120849 & 19399 & 51328 & 20268 & 87354 & 23941 & 55909 & 40003 & 12495 & 12871 & 6271 \\
\hline Percentual & $26,81 \%$ & $4,30 \%$ & $11,39 \%$ & $4,50 \%$ & $19,38 \%$ & $5,31 \%$ & $12,40 \%$ & $8,88 \%$ & $2,77 \%$ & $2,86 \%$ & $1,39 \%$ \\
\hline
\end{tabular}

Tabela 4A. Raça dos pacientes com tuberculose na Região de Saúde Centro-Sul (RJ) entre 2015 e 2019.

\begin{tabular}{ccccccc}
\hline \multicolumn{5}{c}{ Região de Saúde Centro-Sul (RJ) } \\
\hline Raça & Ignorado & Branca & Preta & Amarela & Parda & Indígena \\
Pacientes & 178 & 209 & 174 & 11 & 168 & 1 \\
Percentual & $24,02 \%$ & $28,20 \%$ & $23,48 \%$ & $1,48 \%$ & $22,67 \%$ & $0,13 \%$ \\
\hline & Fonte: Ministério da Saúde - Sistema de Informação de Agravos e Notificação - Sinan Net.
\end{tabular}

Tabela 4B. Raça dos pacientes com tuberculose no Brasil entre 2015 e 2019.

\begin{tabular}{ccccccc}
\hline \multicolumn{7}{c}{ Brasil } \\
\hline Raça & Ignorado & Branca & Preta & Amarela & Parda & Indígena \\
Pacientes & 34619 & 134348 & 57647 & 3277 & 216109 & 4687 \\
& & & & & 47,95 & $1,04 \%$ \\
\hline
\end{tabular}

Fonte: Ministério da Saúde - Sistema de Informação de Agravos e Notificação - Sinan Net.

Tabela 5A. Sexo dos pacientes com tuberculose na Região de Saúde Centro-Sul (RJ) entre 2015 e 2019.

\begin{tabular}{lccc}
\hline & \multicolumn{4}{c}{ Região de Saúde Centro-Sul (RJ) } \\
\hline Sexo & Ignorado & Masculino & Feminino \\
Pacientes & 0 & 516 & 225 \\
Percentual & $0 \%$ & $69,64 \%$ & $30,36 \%$ \\
\hline
\end{tabular}

Fonte: Ministério da Saúde - Sistema de Informação de Agravos e Notificação - Sinan Net.

amarela e indígena. No Brasil a raça parda foi a de maior percentual, com $47,95 \%$ das notificações, conforme Tabela 4B.

Vale ressaltar que o termo "raça" indica a noção vulgar nativa de raça, uma vez que não é possível definir geneticamente as raças humanas. São traços fisionômicos de fenótipos e genótipos que não possuem respaldo científico, pois as raças são uma construção social, entrando no campo da cultura e da cor da pele ${ }^{6}$.

Em relação ao sexo, a Tabela $5 \mathrm{~A}$ mostra que há uma diferença muito grande na Região de Saúde
Centro-Sul (RJ), onde $69,64 \%$ dos casos são em pessoas do sexo masculino, contra $30,36 \%$ de pessoas do sexo feminino, tornando um número aproximadamente de 2:1 de casos do sexo masculino para caso do sexo feminino. Importante ressaltar que não houve nenhum caso em que tal informação deixou de ser apontada nas 741 notificações existentes. No Brasil essa proporção foi praticamente a mesma, com $69,667 \%$ dos pacientes sendo do sexo masculino, conforme Tabela 5B.

A Tabela 6A mostra o tipo de entrada como notificação de caso na Região de Saúde Centro-Sul 
Tabela 5B. Sexo dos pacientes com tuberculose no Brasil entre 2015 e 2019.

\begin{tabular}{lccc}
\hline & \multicolumn{3}{c}{ Brasil } \\
\hline Sexo & Ignorado & Masculino & Feminino \\
Pacientes & 31 & 313983 & 136674 \\
Percentual & $0,007 \%$ & $69,667 \%$ & $30,326 \%$ \\
\hline
\end{tabular}

Fonte: Ministério da Saúde - Sistema de Informação de Agravos e Notificação - Sinan Net.

Tabela 6A. Tipo de Entrada dos casos de tuberculose na Região de Saúde Centro-Sul (RJ) entre 2015 e 2019.

\begin{tabular}{lcccccc}
\hline \multicolumn{7}{c}{ Região de Saúde Centro-Sul (RJ) } \\
\hline Entrada & Caso novo & Recidiva & Reingresso & Não sabe & Transferência & Pós-óbito \\
Pacientes & 609 & 70 & 50 & 3 & 9 & 0 \\
Percentual & $82,19 \%$ & $9,45 \%$ & $6,75 \%$ & $0,40 \%$ & $1,21 \%$ & $0,00 \%$ \\
\hline
\end{tabular}

Fonte: Ministério da Saúde - Sistema de Informação de Agravos e Notificação - Sinan Net.

(RJ). Os casos novos são $82,19 \%$, de recidivas $9,45 \%$ e reingresso $6,75 \%$. Baixos valores de transferência $1,21 \%$ e que não souberam informar $0,40 \%$. Não houve nenhum caso descoberto depois do óbito. No Brasil também há como principal entrada a notificação de casos novos, com 80,14\%, conforme Tabela 6B.

Em relação à forma, a Tabela 7A mostra que 89,61\% dos casos da Região de Saúde Centro-Sul (RJ) foram da forma pulmonar, $8,10 \%$ foram da forma extrapulmonare $2,29 \%$ foram das duas simultaneamente, pulmonar e extrapulmonar. No Brasil também houve maior percentual da forma pulmonar, com $84,54 \%$ das notificações, conforme Tabela 7B.

Em relação à presença do vírus $\mathrm{HIV} \mathrm{e}$ tuberculose no mesmo paciente, a Tabela $8 \mathrm{~A}$ revela que há um percentual de 6,88\% na Região de Saúde

Tabela 6B. Tipo de Entrada dos casos de tuberculose no Brasil entre 2015 e 2019.

\begin{tabular}{lrrrrrr}
\hline \multicolumn{5}{c}{ Brasil } \\
\hline Entrada & Caso novo & Recidiva & Reingresso & Não sabe & Transferência & Pós-óbito \\
Pacientes & 361192 & 33147 & 38873 & 1607 & 12829 & 3040 \\
Percentual & $80,14 \%$ & $7,35 \%$ & $8,63 \%$ & $0,36 \%$ & $2,85 \%$ & $0,67 \%$ \\
\hline
\end{tabular}

Fonte: Ministério da Saúde - Sistema de Informação de Agravos e Notificação - Sinan Net.

Tabela 7A. Forma dos casos de tuberculose na Região de Saúde Centro-Sul (RJ) entre 2015 e 2019.

\begin{tabular}{lcccc}
\hline \multicolumn{4}{c}{ Região de Saúde Centro-Sul (RJ) } \\
\hline Forma & Ignorado & Pulmonar & Extrapulmonar & Pulmonar + Extrapulmonar \\
Pacientes & 0 & 664 & 60 & 17 \\
Percentual & $0,00 \%$ & $89,61 \%$ & $8,10 \%$ & $2,29 \%$ \\
\hline & Fonte: Ministério da Saúde - Sistema de Informação de Agravos e Notificação - Sinan Net.
\end{tabular}

Tabela 7B. Forma dos casos de tuberculose no Brasil entre 2015 e 2019.

\begin{tabular}{lcccc}
\hline \multicolumn{4}{c}{ Brasil } \\
\hline Forma & Ignorado & Pulmonar & Extrapulmonar & Pulmonar + Extrapulmonar \\
Pacientes & 219 & 381016 & 55931 & 13522 \\
Percentual & $0,05 \%$ & $84,54 \%$ & $12,41 \%$ & $3,00 \%$ \\
\hline
\end{tabular}


Tabela 8A. Pacientes com tuberculose e infecção pelo HIV na Região de Saúde Centro-Sul (RJ) entre 2015 e 2019.

\begin{tabular}{cccccc}
\hline \multicolumn{5}{c}{ Região de Saúde Centro-Sul (RJ) } \\
\hline Diagnóstico & Ignorado & Positivo & Negativo & Em andamento & Não realizado \\
Pacientes & 0 & 51 & 621 & 17 & 52 \\
Percentual & $0,00 \%$ & $6,88 \%$ & $83,81 \%$ & $2,29 \%$ & $6,88 \%$ \\
\hline & Fonte: Ministério da Saúde - Sistema de Informação de Agravos e Notificação - Sinan Net.
\end{tabular}

Centro-Sul (RJ). Além disso, há quantidade significante de pacientes com o diagnóstico e sem o teste de HIV realizado, um total de 52 pacientes, totalizando $6,88 \%$. No Brasil esse percentual foi de $10,72 \%$, conforme Tabela 8B.

A Tabela 9A mostra os resultados em relação ao tratamento supervisionado na Região de Saúde Centro-Sul (RJ), há um número muito elevado de pacientes em que esta informação foi ignorada, $32,93 \%$. Há também um número ainda maior de pacientes que não realizaram o tratamento supervisionado, 59,92\%. Logo, apenas $7,15 \%$ dos pacientes realizaram o tratamento supervisionado. No Brasil esse percentual foi muito superior, sendo $34,06 \%$ dos tratamentos sendo supervisionados, conforme Tabela 9B.

Em relação à situação de encerramento a Tabela $10 \mathrm{~A}$ revela que $12,28 \%$ dos pacientes da Região de Saúde Centro-Sul (RJ) tiveram a informação ignorada na notificação, $65,05 \%$ foram curados e $11,74 \%$ abandonaram o tratamento. Um total de $2,02 \%$ dos pacientes faleceu por tuberculose no período e 5,67\% dos pacientes faleceram por outras causas. $\mathrm{O}$ número de pacientes com tuberculose drogarresistente foi de 3, totalizando $0,40 \%$ dos casos. No Brasil o percentual de curados foi um pouco menor, $60,98 \%$, conforme Tabela 10B.

Tabela 8B. Pacientes com tuberculose e infecção pelo HIV no Brasil entre 2015 e 2019.

\begin{tabular}{cccccc}
\hline & \multicolumn{5}{c}{ Brasil } \\
\hline Diagnóstico & Ignorado & Positivo & Negativo & Em andamento & Não realizado \\
Pacientes & 1918 & 48316 & 315731 & 8352 & 76371 \\
Percentual & $0,43 \%$ & $10,72 \%$ & 70,06 & $1,85 \%$ & $16,95 \%$ \\
\hline
\end{tabular}

Fonte: Ministério da Saúde - Sistema de Informação de Agravos e Notificação - Sinan Net.

Tabela 9A. Tratamento supervisionado dos casos de tuberculose na Região de Saúde Centro-Sul (RJ) entre 2015 e 2019.'

\begin{tabular}{cccc}
\hline & \multicolumn{4}{c}{ Região de Saúde Centro-Sul (RJ) } \\
\hline Tratamento supervisionado & Ignorado & Sim & Não \\
Pacientes & 244 & 53 & 444 \\
Percentual & $32,93 \%$ & $7,15 \%$ & $59,92 \%$ \\
\hline
\end{tabular}

Fonte: Ministério da Saúde - Sistema de Informação de Agravos e Notificação - Sinan Net

Tabela 9B. Tratamento supervisionado dos casos de tuberculose no Brasil entre 2015 e 2019.

\begin{tabular}{cccc}
\hline & \multicolumn{3}{c}{ Brasil } \\
\hline Tratamento supervisionado & Ignorado & Sim & Não \\
Pacientes & 138681 & 153498 & 158509 \\
Percentual & $30,77 \%$ & $34,06 \%$ & $35,17 \%$ \\
\hline
\end{tabular}

Fonte: Ministério da Saúde - Sistema de Informação de Agravos e Notificação - Sinan Net. 
Tabela 10A. Situação de encerramento dos casos de tuberculose na Região de Saúde Centro-Sul (RJ) entre 2015 e 2019.

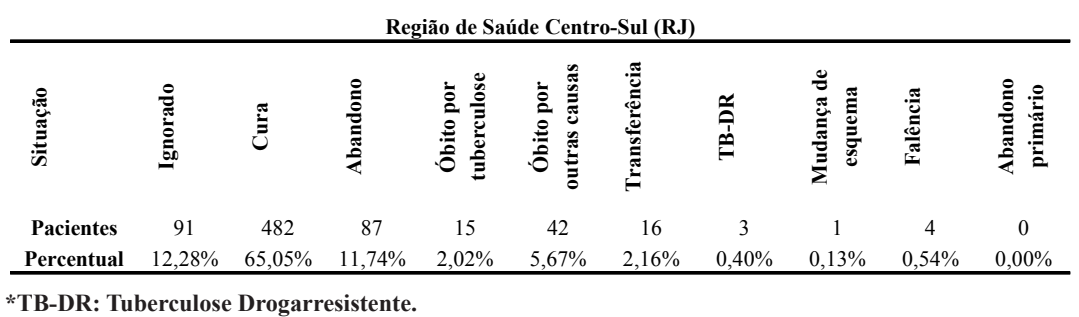

Fonte: Ministério da Saúde - Sistema de Informação de Agravos e Notificação - Sinan Net.

Tabela 10B. Situação de encerramento dos casos de tuberculose no Brasil entre 2015 e 2019 .

\begin{tabular}{|c|c|c|c|c|c|c|c|c|c|c|}
\hline \multicolumn{11}{|c|}{ Brasil } \\
\hline 胥 & 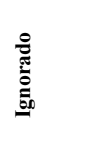 & 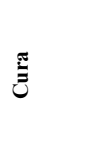 & 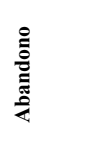 & 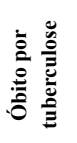 & 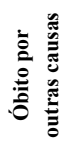 & 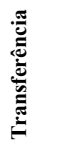 & 蓄 & 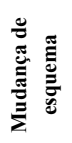 & 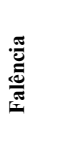 & 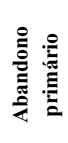 \\
\hline Pacientes & 51965 & 274819 & 50483 & 15509 & 18629 & 28421 & 4817 & 2552 & 331 & 3162 \\
\hline Percentual & $11.53 \%$ & $60,98 \%$ & $11,20 \%$ & $3,44 \%$ & $4,13 \%$ & $6,30 \%$ & $1,07 \%$ & $0,57 \%$ & $0,07 \%$ & $0,70 \%$ \\
\hline
\end{tabular}

Fonte: Ministério da Saúde - Sistema de Informação de Agravos e Notificação - Sinan Net.

\section{Discussão}

Em busca da diminuição dos novos casos e da mortalidade da tuberculose no Brasil, o Ministério da Saúde criou em 2017, o Plano Nacional pelo Fim da Tuberculose como Problema de Saúde Pública. Para isso, ações de prevenção são pensadas em nível nacional e em nível local. A utilização do perfil epidemiológico é necessária para a criação e implementação de estratégias para a redução de casos e da mortalidade da tuberculose de forma local. A redução do coeficiente de incidência e mortalidade vem com estratégias efetivas de prevenção, diagnóstico e tratamento eficaz da doença. Através do traçado do perfil epidemiológico da doença na Região Centro-Sul (RJ) será possível, posteriormente, traçar medidas que visem reduzir novos casos, identificar quais os indivíduos mais vulneráveis na região e principais motivos de abandono do tratamento ${ }^{3}$.

O Estado do Rio de Janeiro possuía em 2017, o maior coeficiente de mortalidade do Brasil, sendo cinco óbitos por tuberculose/100.000 habitantes ${ }^{3}$, entretanto a Região de Saúde Centro-Sul compõe apenas $2 \%$ da população do Estado, com 254.103 habitantes ${ }^{7}$.

Apesar da existência da vacina BCG, esta não é ainda uma vacina ideal, embora tenha um papel importante para o controle da doença, principalmente onde há elevada carga de tuberculose. A vacina BCG protege contra a ocorrência de formas graves, como a tuberculose miliar e a meningite tuberculosa, principalmente em crianças ${ }^{8}$.

Para traçar o perfil epidemiológico da Região de Saúde Centro-Sul (RJ), a primeira característica foi a faixa etária. Assim como os dados do Brasil, a faixa etária com maior percentual foi a de 20 a 39 anos e a segunda com maior percentual foi a de 40 a 59 anos. Tamanha foi a diferença para as demais faixas, que se pode considerar que a faixa etária com maior incidência de tuberculose foi entre 20 a 59 anos, poupando extremos de idade, ou seja, menores de 20 anos e maiores de 59 anos, que possuem somadas apenas $26 \%$ dos casos à nível de Região Centro-Sul (RJ) e 22,72\% à nível de Brasil.

O diagnóstico de tuberculose em crianças é mais complexo que em adultos, uma vez que não há um exame considerado como padrão-ouro que o torne direto 9 . Portanto, é utilizado hoje no Brasil um sistema de pontuação com o quadro clínico e radiológico, contato com adultos com tuberculose, prova tuberculínica e estado nutricional da criança. Em virtude dessa dificuldade, em muitos casos pode haver subnotificação de casos em crianças, tendo o médico papel fundamental na suspeita do diagnóstico e no uso correto do sistema de pontuação ${ }^{10}$. O diagnóstico em idosos também é complexo, que, somado à existência de outras doenças pré-existentes, determina maior mortalidade para esta faixa etária ${ }^{11}$.

Em relação à escolaridade faltam informações 
importantes, pois há, particularmente na Região CentroSul (RJ), uma quantidade muito grande de notificações nas quais esta não foi informada, $61,40 \%$. Em nível nacional a quantidade de notificações em branco quanto à escolaridade é menor, mas ainda sim um número muito relevante, $26,81 \%$. Dessa forma, na Região Centro-Sul (RJ) o maior número de pacientes com tuberculose possui até o ensino médio completo, diferenciando do Brasil, que possui maior percentual de pacientes com tuberculose com a $5^{\text {a }}$ a $8^{\text {a }}$ série incompleta. Assim, embora haja uma quantidade muito grande de dados faltantes para a devida comparação, a Região CentroSul (RJ) possui mais pacientes com escolaridade um pouco maior que no restante do Brasil, ou seja, com ensino médio completo e menor quantidade de pacientes com quase nenhuma escolaridade, como analfabetos, $1^{\mathrm{a}}$ a $4^{\mathrm{a}}$ série incompleta e $4^{\mathrm{a}}$ série completa ${ }^{12}$. A baixa escolaridade é um indicador que contribui para o aumento de casos, abandono ao tratamento e recidivas, ficando mais expostos e vulneráveis à tuberculose, sendo responsáveis pelo aumento da incidência e da menor adesão ao tratamento ${ }^{13}$.

Quanto à raça/cor na Região de Saúde CentroSul (RJ) foi identificado um quantitativo de notificações, no período pesquisado, extremamente amplo. Mesmo assim, a raça com maior percentual com diagnóstico de tuberculose na Região Centro-Sul (RJ) é a raça branca $(28,20 \%)$, embora a diferença para raça preta $(23,48 \%)$ e para raça parda $(22,67 \%)$ seja muito pequena. As raças amarela $(1,48 \%)$ e indígena $(0,13 \%)$ aparecem em quantidade muito menor. Em nível Brasil a raça mais comum é a raça parda (47,95\%), seguida da raça branca $(29,81 \%)$ e menor valor a raça preta $(12,79 \%)$.

Vale ressaltar, mais uma vez, que o conceito de raça humana está ultrapassado, embora haja na notificação da tuberculose tal campo para preenchimento ao invés de cor da pele ${ }^{6}$.

Em relação ao sexo, os percentuais da Região Centro-Sul (RJ) e do Brasil são semelhantes, com $69,64 \%$ e $69,667 \%$, respectivamente, de pacientes do sexo masculino. Vale ressaltar que em todas as notificações da Região Centro-Sul essa informação foi preenchida e, em todo o Brasil, apenas 31 pacientes não tiveram essa informação preenchida na notificação. Essa maior quantidade de casos de pacientes do sexo masculino possui relação com estigmas relacionados ao processo saúde-doença e a relutância à aceitação da doença pelos homens, motivados na crença de não adoecimento, bem como a não procura por atendimento ou falta de conhecimento da doença ${ }^{14}$.

No que diz respeito à forma de entrada, os dados da Região Centro-Sul (RJ) também são muito similares aos do Brasil, sendo a maioria de casos novos da doença. Percentual um pouco maior de casos de recidiva na Região Centro-Sul (RJ) em relação ao restante do Brasil. Portanto, a principal preocupação deverá ser com diminuição dos casos novos e tentativa de diminuição de recidivas. Poucas foram as situações em que a entrada se deu pós-óbito no Brasil e nenhuma na Região Centro-Sul (RJ).

Quanto à forma de tuberculose, os percentuais da Região Centro-Sul se assemelham aos do Brasil, com quase $90 \%$ dos casos sendo da forma pulmonar. Embora minoria, os casos de tuberculose extrapulmonar ou concomitantemente com a pulmonar continuam elevados, mesmo após a instituição da vacina BCG no Brasil em 1927, que protege contra as formas graves como a meningite tuberculose e a tuberculose miliar. Essa ainda alta incidência de formas extrapulmonares pode estar relacionada à abrangência da vacinação, à efetividade da vacinação ou à comorbidades próprias dos pacientes ${ }^{15}$.

Em relação à presença concomitante do vírus HIV e da tuberculose na Região Centro-Sul, há 6,88\% dos casos notificados, contra $10,72 \%$ dos casos notificados no Brasil. Devem-se intensificar, entretanto, os testes para HIV nos casos notificados, uma vez que pode haver um número ainda maior de pacientes coinfectados e sem o diagnóstico de HIV. O Programa Nacional de Combate da Tuberculose prevê a disponibilização de testes antiHIV para $100 \%$ dos adultos com tuberculose ${ }^{16}$. As principais características dos pacientes com infecção concomitante HIV/tuberculose são: sexo masculino, idade economicamente ativa, baixa escolaridade e heterossexualidade ${ }^{17}$.

O tratamento supervisionado deve estar disponível em $100 \%$ das unidades de saúde da atenção primária, de forma a horizontalizar o tratamento da tuberculose. Além disso, pelo menos $80 \%$ dos pacientes bacilíferos devem receber o tratamento supervisionado, de acordo com o Programa Nacional de Controle da Tuberculose ${ }^{16}$. Sendo assim, observa-se que na Região Centro-Sul (RJ) há quase $60 \%$ dos pacientes sem o tratamento supervisionado e quase $33 \%$ dos pacientes com essa informação ignorada na notificação. Esse percentual de pacientes que receberam o tratamento supervisionado está muito inferior ao número nacional de $35,17 \%$, evidenciando que há necessidade de intensificação do tratamento supervisionado de forma regional.

O paciente com tuberculose está considerado curado quando há pelo menos duas baciloscopias negativas, uma ainda na fase de acompanhamento e a segunda na fase final do tratamento ${ }^{18}$. Nesse ponto a Região Centro-Sul (RJ) apresentou um índice de cura de $65,05 \%$, maior que índice nacional no período que foi de $60,98 \%$. Os percentuais de pacientes com tal informação ignorada na notificação e de abandono do tratamento foram similares aos níveis nacionais. Também foi menor o percentual de pacientes com tuberculose drogarresistente, com número 2,6 vezes menor $(1,07 \% / 0,40 \%)$. 
Em estudo realizado no município de São Carlos (SP) em 2017, o perfil encontrado foi o de pacientes entre 15 a 39 anos $(39,5 \%), 4$ a 7 anos de escolaridade (30,27\%), sexo masculino $(57,3 \%)$, tipo de entrada como um caso novo $(72,03 \%)$, forma pulmonar $(72,41 \%)$, sem infecção concomitante com o HIV (75\%), realização do tratamento supervisionado $(87,63 \%)$ e encerramento do caso como cura $(77,6 \%)$. Não houve a inclusão da variável cor/raça no estudo. $\mathrm{O}$ percentual de casos com infecção concomitante com o HIV foi de $14,05 \%{ }^{19}$. A maior parte das variáveis segue o mesmo perfil encontrado na região, exceto pela cor/raça e a realização do tratamento supervisionado. A cor/raça não foi incluída no estudo. A realização do tratamento supervisionado está inferior na Região Centro-Sul (RJ), embora haja quantidade muito grande de respostas ignoradas, $32,93 \%$, contra apenas $0,67 \%$ no município de São Carlos (SP).

Em outro estudo realizado no Estado do Amazonas, em 2016 que comparou o perfil epidemiológico dos pacientes que eram um caso novo de tuberculose com pacientes que abandonaram o tratamento, o percentual de pacientes do sexo masculino também foi elevado para ambas as situações, novo caso $(58,3 \%)$ e retratamento $(66,7 \%)$. A cor/raça mais encontrada foi a parda, com $67,9 \%$ para casos novos e $69 \%$ para retratamento. O estudo concluiu que não foi possível verificar associação da necessidade de retratamento por abandono com características clínicoepidemiológicas, embora tenham participado apenas 126 pacientes $^{12}$.

Em 2017, foi realizado um estudo semelhante no município de São Paulo (SP) em que, das variáveis presentes, a forma pulmonar também foi a de maior incidência $(76,4 \%)$, a situação de encerramento foi de cura $(77,0 \%)$, a maioria dos pacientes realizaram o teste para HIV $(84,7 \%)$ e a maioria dos pacientes realizou o tratamento supervisionado (60,5\%). Embora na Região Centro-Sul (RJ) o percentual de não realização tenha sido menor $(6,88 \%$ contra $15,3 \%)$, são demandadas ações que visem chegar esse número ao mais próximo possível de $100 \%$, devido à importância do diagnóstico da pessoa com infecção concomitante de tuberculose e HIV. O percentual de pacientes que receberam o tratamento supervisionado foi muito similar, sendo $59,92 \%$ na Região Centro-Sul (RJ) e $60,5 \%$ no município de São Paulo (SP) ${ }^{20}$.

\section{Conclusão}

Diante à gravidade da situação da tuberculose no Brasil, o estudo analisou dados dos pacientes portadores de tuberculose na Região de Saúde Centro-Sul (RJ), ficando evidente a permanência do crescente número de casos de tuberculose em nosso meio.
Quanto ao preenchimento das fichas do SINAN foi identificada no estudo uma quantidade de casos notificados que tiveram informações extremamente relevantes ignoradas e não informadas, necessitando assim de um melhor preenchimento pelos profissionais que prestam a assistência, considerando que a atenção primária em saúde é o primeiro acesso e saída dos usuários do Sistema Único de Saúde.

Os resultados deste estudo encontraram como perfil epidemiológico mais comum de pacientes com a doença as seguintes características: escolaridade como ensino médio completo, raça branca, sexo masculino, entrada como um novo caso, forma pulmonar, positividade para HIV menor que o índice nacional e com maior percentual de teste, menor índice de realização de tratamento supervisionado e maior índice de encerramento por cura.

O perfil mais comum encontrado foi similar ao de estudos realizados em outras cidades e regiões do Brasil. A maior discrepância para a região do estudo foi a realização do tratamento supervisionado para apenas $7,15 \%$ dos pacientes com tuberculose.

Existem ações específicas que podem ser implementadas para melhorar os índices expostos como intensificação das visitas domiciliares e busca ativa para interromper a cadeia de transmissão, redução do tempo de diagnóstico e início do tratamento, intensificação do tratamento supervisionado, identificação precoce de sintomáticos respiratórios, controle de contatos e criação de vínculo com a comunidade.

Por todas as variáveis analisadas, há ainda muito a melhorar na Região de Saúde Centro-Sul (RJ), embora esteja com números superiores aos índices nacionais para a maioria das variáveis analisadas no estudo.

Por último, por se tratar de um estudo que utilizou como base dados secundários das notificações, há uma limitação pela possibilidade de subnotificações e, como visto, pela ausência de preenchimento de algumas informações.

\section{Referências}

1. Tavares W, Marinho LAC. Rotinas de Diagnósticos e Tratamento das Doenças Infecciosas e Parasitárias. 2015. 1057 p.

2. Brasil. Boletim Epidemiológico de Tuberculose. Ministério da Saúde Secr Vigilância em Saúde Dep Doenças Condições Crônicas e Infecções Sex Transm. 2020;1:9.

3. Brasil. Brasil Livre da Tuberculose: Plano Nacional pelo Fim da Tuberculose como Problema de Saúde Pública [Internet]. Secretaria de Vigilância em Saúde. Departamento de Vigilância das Doenças Transmissíveis. 2017. 52 p. Available from: ttp://bvsms.saude.gov.br/bvs/ publicacoes/brasil livre tuberculose plano nacional.pdf

4. Arruda KG de. Avalição dos custos do tratamento de tuberculose em município de médio porte do nordeste brasileiro. 2014;58. Available from: http://www.repositorio.ufpe.br/handle/123456789/11539

5. Chrispim PPM. Uma contribuição para a discussão sobre avaliações de programas de controle da tuberculose. 2011;138. 
6. Sansone L, Araujo Pinho O. Raça - Novas perspectivas antropológicas. 2008. 63 p.

7. Brasil. $1^{\circ}$ Seminário de Gestão Participativa em Saúde das Regiões Médio-Paraíba, Centro-Sul e Baía da Ilha Grande do Rio de Janeiro [Internet]. Secretaria de Gestão Estratégica e Participativa. 2007. 80 p. Available from: http://bvsms.saude.gov.br/bvs/publicacoes/07_0156_M.pdf

8. Pereira SM, Dantas OMS, Ximenes R, Barreto ML. Vacina BCG contra tuberculose: efeito protetor e políticas de vacinação. Rev Saude Publica. 2007;41(suppl 1):59-66.

9. Cano APG, Romaneli MTN, Pereira RM, Tresoldi AT. Tuberculose Em Pacientes Pediátricos: Como Tem Sido Feito O Diagnóstico? Rev Paul Pediatr. 2017;35(2):165-70.

10. Maciel ELN, Dietze R, Silva RECDF, Hadad DJ, Struchiner CJ. Avaliação do sistema de pontuação para o diagnóstico da tuberculose na infância preconizado pelo Ministério da Saúde, Brasil. Cad Saude Publica. 2008;24(2):402-8.

11. Helena S, Vendramini F, Isabel R, Gonzales C, Monroe AA. Tuberculose No Idoso : Análise Do Conceito Tuberculosis in the Elderly: Concept Analysis Tuberculosis En El Anciano : Análisis De Concepto. Rev Latino-am Enferm. 2003;11(1):96-103.

12. Belchior A de S, Arcêncio RA, Mainbourg EMT. Differences in the clinical-epidemiological profile between new cases of tuberculosis and retreatment cases after default. Rev da Esc Enferm. 2016;50(4):619-25.

13. Silva CCAV, Andrade MS, Cardoso MD. Fatores associados ao abandono do tratamento de tuberculose em indivíduos acompanhados em unidades de saúde de referência na cidade do Recife, Estado de Pernambuco, Brasil, entre 2005 e 2010. Epidemiol e Serviços Saúde. 2013;22(1):77-85.

14. Souza Júnior EV de, Nunes GA, Cruz DP, Boery EN, Boery RNS de $\mathrm{O}$. Internações hospitalares e impacto financeiro por tuberculose pulmonar na Bahia, Brasil. Enfermería actual en Costa Rica. 2018;18(35).

15. Oliva HNP, Oliveira AG, Gondinho ACVC de Q, Nunes FM. Incidência de tuberculose extrapulmonar. Soc Bras Clínica Medica. 2019;17(2):63-5.

16. BRASIL. Programa Nacional de Controle de Tuberculose. Secretária vigilância em saúde. 2004;1-28.

17. Bastos SH, Taminato M, Fernandes H, Figueiredo TMRM de, Nichiata LYI, Hino P. Perfil Sociodemográfico e de saúde da coinfecção tuberculose / HIV no Brasil : revisão sistemática. Rev Bras Enferm. 2019;72(5):1458-65.

18. Ministério da Saúde. Brasil. Registro de pacientes e acompanhamento de tratamento dos casos de tuberculose. 2006;26.

19. Fusco APB, Arcêncio RA, Yamamura M, Palha PF, Reis AA Dos, Alecrim TFDA, et al. Spatial distribution of tuberculosis in a municipality in the interior of São Paulo, 2008-2013. Rev Lat Am Enfermagem. 2017;25:2008-13.

20. Pinto PFPS, Silveira C, Rujula MJP, Chiaravalloti Neto F, Ribeiro MCS de A. Perfil epidemiológico da tuberculose no município de São Paulo de 2006 a 2013. Rev Bras Epidemiol. 2017;20(3):549-57. 